\title{
Robustness Analysis of Holonic Multi-Agent Systems: Application to Traffic Signals Control
}

\author{
Monireh ABDOOS
}

\begin{abstract}
Multi-Agent Systems (MASs) provide a powerful tool to model distributed systems. Large-scale systems contain many autonomous agents, and therefore, the agents should be able to work in a group and collaborate toward common objectives. Holonic Multi-Agent Systems (HMASs) present a suitable organization, especially in large-scale systems. The idea behind HMASs is a division of a system into smaller sub-systems in a recurrent way. A holon is defined as a self-similar structure that comprises holons as sub-structures. Therefore, a holarchy is a hierarchy of holons that act as autonomous wholes in super-ordination to their parts and as dependent parts in subordination to controls on higher levels. There are two main attributes for a holarchy, the first attribute ensures that holons are in stable forms, which are robust against disturbances. The second one confirms that the holons are in intermediate forms, which provide the proper functionality for the whole. In this paper, we study the robustness of a holarchy for traffic signals control. Robustness is an essential feature for providing reliable solutions, especially in real world applications. We show that holonic MAS can be effectively used for traffic signals control as a robust modeling method.
\end{abstract}

Keywords: Holonic Multi-Agent Systems; robustness; statistical test; traffic signals control

\section{INTRODUCTION}

Multi-agent System (MAS) is an effective solution for modelling and simulation of large scale distributed systems. MASs generally consist of autonomous, reactive, proactive, and interacting agents working toward a joint goal. The problems which are modeled by multi-agent systems can be considered as decision-making problems where an agent needs to decide which action should be executed in order to maximize its objective function. Optimization of the decision-making process in multiagent systems is very challenging especially in a largescale system because each agent should consider other agents'actions in the system. Organizing agents into a network is a common approach to handle large scale systems, where each agent interacts only with a number of agents [1]. In organization-based systems, interactions among the agents are decreased. Therefore, it is a very common method to reduce the complexity of large systems. In MAS, an organization is defined as a collection of roles, relationships and authority structures that rule the behavior of the multi-agent system [2]. There are numerous models proposed for the concept of organizations in a multi-agent system such as hierarchies, holarchies, coalitions, teams, congregation, societies, federations, markets, matrix organization and formant organizations [2].

Organization-based multi-agent systems have been proposed for traffic signals control many years ago. The hierarchical organization has been presented to control the traffic signals in different kinds of literature. Some related works, which have utilized the hierarchical organization for traffic signals control in agent-based systems, are mentioned in this section. However, the readers are referred to [3] and [4] for a comprehensive survey of applications of agents and multi-agent systems for traffic signals control. Chen and Li proposed the architecture of hierarchical networked traffic signals controllers based on multi-agent systems [5]. Organization, coordination, and execution are three layers incorporated in the control system. On the top of the system, an organization layer provides some services utilizing three databases including control algorithm, typical intersection and agents' database. Although the proposed system in [5] can be effectively used in simulations, the architecture contains many different modules either for online and offline learning in organization layer or in two layers. Moreover, it seems that the implementation and execution of their system are non-trivial and computationally expensive. Decentralized agent-based hierarchical control methods break down the system into smaller subsystems interacting with each other. Choy et al. [6] and Srinivasan et al. [7] also introduced a hierarchical multi-agent system consisting of three layers of agents: controller agents, zone controller agents, and regional controller agents. The implementation of agents is based on feed-forward neural network and fuzzy logic theories. The system also provides online learning capabilities, including reinforcement learning, learning rate, weight adjustment, and dynamic update of fuzzy relations using the evolutionary algorithm, to allow agents to dynamically adapt to changing environments. The hybrid method effectively controls traffic signals in a dynamic environment. However, the system is difficult to extend due to the computational cost of implementation of both the neural network and the evolutionary algorithm.

A hierarchical multi-agent system with three levels has also been presented in [8]. On the first level, local traffic agents represent intersections and coordinator agents stand on the second level. Bazzanet. al have proposed a hierarchical learning method for traffic signals control [9]. Their system is composed of two types of agents: local agents to control intersections and superior agents to control groups of three local agents. Wang developed an agent-based traffic management system in $[10,11]$. Agentbased control breaks down a complex control algorithm into simple task-oriented agents that are distributed over a network. The ability to dynamically deploy and replace control agents as needed allows the network to operate on demand to adapt a variety of control scenarios. The system architecture uses a hierarchical architecture with three levels. The highest level performs the reasoning and planning of task sequences for the control agents; intermediate level dispatches and coordinates control 
agents; and the lowest level hosts and runs the control agents. Control agents are represented by mobile agents that can transfer from remote traffic control centers to field traffic devices or travel from one field device to another $[10,11]$.

Reinforcement Learning (RL) has been widely used for traffic signals control [12-14]. The advantages of using $\mathrm{RL}$ for traffic signals control are: the agents can learn online in a dynamic domain; therefore, they are adaptive to changes in traffic demand. Moreover, RL can be effectively used when no prior knowledge is available [15]. The references $[15,16]$ provide a comprehensive review of the applications of RL to traffic signals control. RL has been used in organization-based MASs in large urban networks in which the agents were organized hierarchically to control the traffic signals in [9, 17]. A large network decomposed to groups of three intersections. The groups were coordinated by a superior agent that recommends a joint action in [9]. A two-level hierarchical MAS to control the traffic signals based on RL was also presented in [17]. The agents at the two levels use RL independently. At first level, each agent controls an intersection by its own local view while its autonomy is restricted by superior agents that stand at the second level to control a region composed of multiple intersections [17].

The holonic organization has been used in $[18,19]$ for traffic signals control. In this study, we focus on holarchies and holonic multi-agent systems; therefore, we describe them in more details in the following sections.

In 1967, A. Koestler invented the term holon [20]. A holon denotes a whole-part construction that can be considered as a component of a higher level system or as a whole consisting of other holons as substructures. The concept of holonic system has been implemented in a programming language [21] and it has been widely used in industrial applications as Holonic Manufacturing Systems $[22,23]$. The holonic multi-agent system has proven to be an effective solution to several problems associated with hierarchical and self-organizing constructions [24] and has been successfully applied to a wide range of complex systems. For instance, we can imply the operations which have been done in transportation [19], distributed sensor management [25], adaptive mesh problem [26], supply chain management [27], health organizations [28], biological network simulation [29], complex software systems [30] and for smart grid market to manage electricity agents in Smart grid operation [31].

The holonic organization provides a powerful tool for intelligent transportation systems (ITS). It has been applied in different applications including pedestrian simulation [32], global road transportation system [33], transport scheduling [34] as different aspects of ITS.

Although holonic organization is a hierarchical nested structure, it differs from hierarchy [2]. According to definitions, the main characteristic of a holonic system is that the holons are self-similar and partially autonomous. Each holon consists of one or more subordinate holons and can be a member of one or more superordinate holons. Therefore, it keeps both features of recursion and hierarchical structure.

Holonic multi-agent system characteristics will be discussed in the next section. In this paper, we introduce a holonic MAS for traffic signals control and study the robustness of the method. It will be verified that the holonic organization accommodates a robust tool against internal and external disturbances while it improves the efficiency. The rest of the current paper is as follows: Section 2 includes the properties of a holonic multi-agent system. Robustness concept in HMAS is presented in Section 3. The proposed HMAS for traffic signals control is introduced in Section 4. Section 5 includes experimental results. Conclusion and future works are given in Section 6.

\section{HOLONIC MULTI-AGENT SYSTEMS}

According to the study conducted by Koestler, a holarchy is defined as a hierarchy of self-regulating holons which act as autonomous wholes in super-ordination to their parts, and also operate as dependent parts in subordination to controls on higher levels as well as in coordination with their local environment. Therefore, a holarchy can be modeled using whole-part relationships. Wholes and parts on any level of the hierarchy are referred as holons. A set of rules defining holonic systems, has been proposed by Koestler, and called Open Hierarchical Systems (OHS) [20] as follows:

- A holonic system has an abstract structure. Indeed, it can be considered as a set of interwoven hierarchies.

- A holon is taken into account both as a part of the system and as a whole.

- A holon follows specific principles, but it can accept different strategies as needed.

- Complex behaviours are situated at the top of the hierarchy while the simple and reactive behaviours are founded at the bottom of the holarchy.

- Communications must follow the hierarchy and along with the direction, should be filtered or detailed.

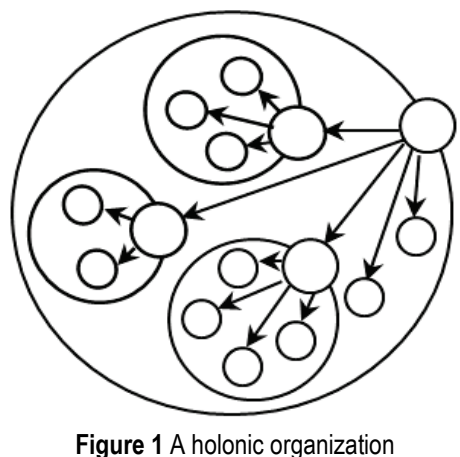

Therefore, a holonic multi-agent system has a hierarchical and flexible structure. They integrate to form a whole while each one can be broken down into holons, which called the recursive breakdown of a problem as shown in Fig. 1. Koestler indicates that holons are autonomous self-reliant units, while they are partially independent to handle contingencies without requesting directions from higher authorities. At the same time, holons can be controlled by (multiple) higher authorities. The first feature ensures that holons are stable forms, which are robust against disturbances. The second feature means that they are intermediate forms providing the appropriate functionality for the super-ordinated whole. 
Holons have some important features that make them well suited for complex and/or distributed systems modeling. The advantages of a holonic system are [35]:

- Scalability: Each holon is autonomous and it can work with little or no knowledge about the other holons. Therefore, we can add more holons to the system without affecting previously existing holons.

- Robustness: Robustness is a result of autonomous nature of a holon. We can remove holons as we can add them to a system without affecting the functionality of other holons as a whole.

- Simplicity of control: Each holon has a simple task to accomplish and it needs a simple control mechanism which can be developed easily compared to centralized systems.

Holons have some disadvantages in comparison with centralized systems [35]:

- Common resources: The autonomous feature of a holon causes that it takes the shared resources without considering other holons. Therefore, it may limit the ability of other holons to work in the system.

- Losing their way: It also comes from the autonomous feature of a holon. A holon can conduct activities that do not contribute to the global goal of the system. They can even conduct activities that are contrary to the global goal.

In this paper, traffic signals controllers have been modelled using a holonic multi-agent system. Among different properties of an HMAS, we concentrate on the robustness of the proposed system. It will be shown that the holonic model is robust against changing the environment and also against the noise.

\section{ROBUSTNESS IN HOLONIC MULTI-AGENT SYSTEMS}

In a MAS, there are multiple agents collaborating towards a goal. The agents need to exchange information over a network for effective cooperation. A MAS must, therefore, take into account the network errors and noises. The theory of cooperative MAS should address the robustness against the noises and uncertainties in the communications. Communication error in the transferred data was considered in [36] for a leader-follower consensus. Failure detection and management have been studied in different kinds of literature. Providing a robust solution against failure was presented in [37] in a manufacturing system by pre0scheduling policies. Uncertain channels have also been considered in [38] and [39]. The robustness of a MAS for collaboration between termites has been studied in [40]. A fault-tolerant infrastructure in Cougar MAS introduced in [41] and applied to a large-scale logistics application over 1000 agents.

Robustness is a key characteristic of a system, especially when used in real world applications. Robustness can be defined according to responses to disturbances, having a different severity level, which affects the performance of the system.

Robustness is a necessity for systems in a dynamic environment, which are partly unpredictable and nondeterministic [42]. Although the robustness is defined in different systems such as dynamic systems by control engineers [43] or computer scientists [44] and [45], it is not appropriate for holonic multi-agent systems. In HMAS, robustness means that holons can be able to work in an environment even in the case of environmental conditions variations. Robustness is a measure of the persistence of systems against disturbances where the system behaviour is formed through adaptive dynamics coupled to organizational architecture [46]. A solution for a dynamic system is robust if small disturbances result in a new solution that remains close to the original solution. Disturbances can be considered as small differences made in the actual state of the system.

\subsection{Wilcoxon-Mann-Whitney Test for Robustness Evaluation}

One of the non-parametric methods for comparing groups and studying associations is Wilcoxon-MannWhitney Test. It provides a different and potentially useful perspective regarding how groups compare and how variables are related to each other. The observation is randomly sampled from each group. Assume that $\mathrm{p}$ is the probability that the randomly sampled observation from the first group, $x_{1}$ is less than the randomly sampled observation from the second group, $x_{2}$ as given in Eq. (1). This equation shows a natural method for characterizing how the groups differ [47].

$p=P\left(x_{1}<x_{2}\right)$

The test is based on a direct estimation of $p$. The groups are statistically identical if there is a chance that an observation from the first group is less than an observation from the second.

$H_{0}: p=0.5$

A method for estimation of $p$ is explained by this method, which is a rank-based technique. The method begins by combination of the observations and a single group, writing them in ascending order and then assigning ranks. Let $n_{1}$ and $n_{2}$ be the number of samples in the first and second groups, respectively. The observations get a rank from $\left[1, n_{1}+n_{2}\right]$. Let $S$ be the sum of the ranks associated with the first group.

$S=\sum_{i=1}^{n_{1}} r_{i}$

$p$ is estimated by Eq. (4)

$p=\frac{U}{n_{1} n_{2}}$

In Eq. (4), $U$ is given as follows:

$U=S-\frac{n_{2}\left(n_{2}+1\right)}{2}$ 
$U$ is called the Wilcoxon-Mann-Whitney $U$ statistic [33]. The description of the test is in terms of $U$. If it is assumed that there are no duplicated values and both groups have identical distributions. Variance of $U$ is given by

$\sigma_{u}^{2}=\frac{n_{1} n_{2}\left(n_{1}+n_{2}+1\right)}{12}$

Therefore, the null hypothesis can be tested by:

$Z=\frac{U-\frac{n_{1} n_{2}}{2}}{\sigma_{u}}$

The null hypothesis is rejected if:

$|Z| \geq c$

where $c$ is the $1-\alpha / 2$ quantile of a standard normal distribution. To evaluate the robustness of a holonic system, the difference between the performances of the the system in similar situations is used as an observation. The goal is to determine whether a fixed value of disturbances leads to the identical differences in all situations or not. For example, distribution of behavioural difference is the same in the following two situations: (a) disturbance rate increases from $0 \%$ to $k \%$ and (b) disturbance rate increases from $k \%$ to $2 k \%$ where $k$ is a constant number.

\section{TRAFFIC SIGNALS CONTROL BASED ON HOLONIC ORGANIZATION}

The holonic organization is used to model a network composed of 16 intersections. Each intersection is controlled by an agent. Groups of four intersections are considered as superior agents. Therefore, a two-level holarchy is applied to model the network as shown in Fig. 2.

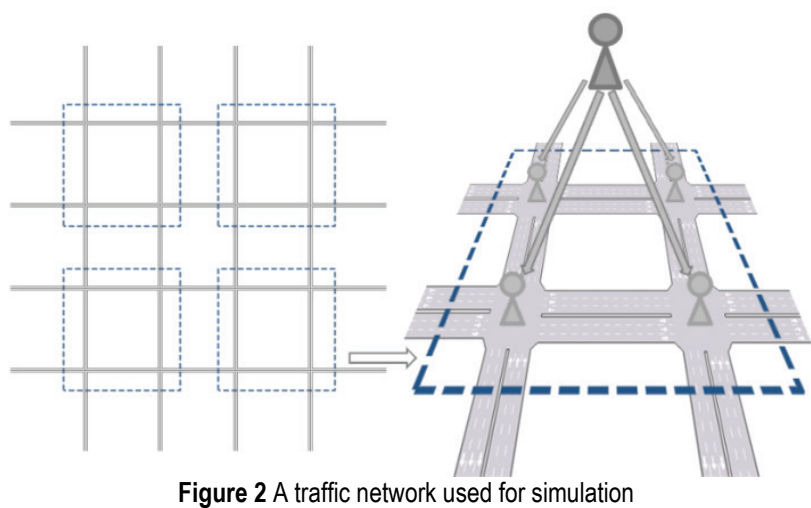

Let call the agents on the bottom level and upper level as sub-holons and super-holons respectively. In this model, there are 16 sub-holons and 4 super-holons that are composed of 4 sub-holons. According to the mentioned rules defined by Koestler for holonic systems, complex activities are situated at the top of the hierarchy. Therefore, a super-holon determines a strategy for its sub-holons. On the other hand, the defining characteristic of a holarchy is the partially-autonomous holon and sub-holons give up a part of their autonomy to their super-holons [2]. A holonic multi-agent system has been proposed for traffic signals control in [19] based on reinforcement learning. We briefly describe the method for traffic signals control in this section. The readers are referred to [19] for more details.

\subsection{Sub-Holons}

A traffic network composed of 16 intersections has been considered in this study, where each intersection is controlled by a sub-holon. In the network configuration, a signal group is assigned for each approaching link. Since all intersections have 4 approaching links, a signal plan containing 4 signal phases is used for all intersections as shown in Fig. 3.

The state space is also defined according to the signal plan [12]. The average queue length of approaching links in a fixed cycle has been utilized to determine the state of the environment. The possible ranked list of them determines the state space. Let $l_{i}$ be the average queue length of the $i^{\text {th }}$ approaching link of an intersection, then $l_{1} \geq l_{2} \geq l_{3} \geq l_{4}$ shows a state in which the average queue length of approaching link $l_{1}$ is the longest among the other links and $l_{4}$ is the shortest one. Two approaching links with the same average queue length ranked considering their order in the signal plan. The number of states is 24 , that equals the number of the permutations of the four lanes, $l_{1}, l_{2}, l_{3}$ and $l_{4}$. The actions are considered by assigning different time splits in a fixed order to the signal groups.

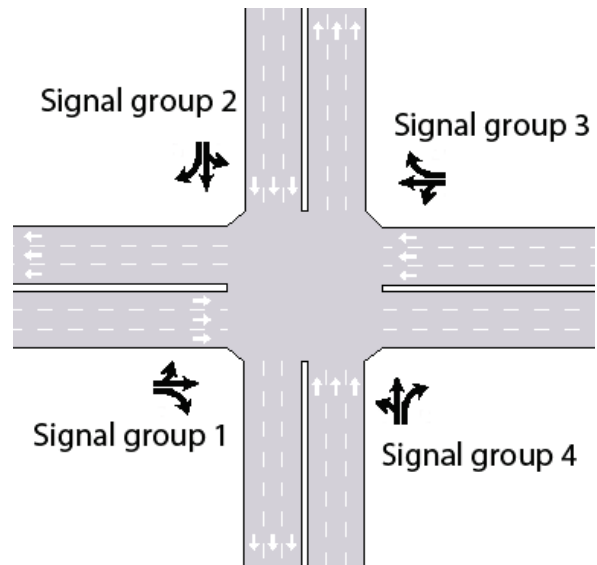

Figure $3 \mathrm{~A}$ signal plan which includes 4 signal groups is used in simulation

It can be mentioned that for a fixed time controller, all available phases should be considered at least once in a cycle. The green time associated to each phase should be at least equal to the time that a stopped vehicle needs to receive a green signal and cross the intersection. To assign cycle length to different phases, the proposed method considers the following steps: first, the minimum green time is assigned to each phase, and then the remaining time is divided into a number of extensions that are assigned to different phases. Assume that minimum green time is computed as 13 seconds. Moreover, there are 4 extensions with fixed length of time, 10 seconds. The actions determine the assignment of 4 extensions to different phases. Let $x_{i}$ show the number of extensions assigned for 
the $i^{\text {th }}$ phase, the possible green time assignment can be formulated as follows [12]:

$$
\sum_{i=1}^{4} x_{i}=4, x_{i} \in\{0,1,2\}
$$

There are 19 possible solutions for Eq. (9). For example, $\left[x_{1}, x_{2}, x_{3}, x_{4}\right]=[0,2,1,1]$ is a solution for Eq. (9) in which the green time assigned to four phases is (13, $33,23,23)$. The reward is inversely proportional to the average queue length of the approaching links, which is normalized to remain between 0 and 1 .

\subsection{Super-Holons}

There are 4 super-holons to control regions which are composed of 4 intersections. A super-holons determine a strategy for the sub-holons. Since sub-holons are partially autonomous holons, super-holons can reduce the size of the action set of sub-holons. Super-holons have a broader view of the network and can prevent the network to be saturated by making a proper decision. Super-holons control the traffic demand and average queue length of the approaching links in the region.

Consider $\eta$ is the maximum density value of approaching links. The state of a super-holon is estimated using $\eta$, as it is shown in Tab. 1 [12].

$$
\eta=\max _{\substack{i=1, \ldots, 4 \\ j=1, \ldots, 4}} \operatorname{density}\left(l_{i, j}\right)
$$

Table 1 The state space of a super-holon

\begin{tabular}{|c|c|} 
Table 1 The state space of a super-holon \\
\hline State 1 & $0 \leq \eta \leq 0.15$ \\
\hline State 2 & $0.15 \leq \eta \leq 0.3$ \\
\hline State 3 & $0.3 \leq \eta \leq 0.45$ \\
\hline State 4 & $0.45 \leq \eta \leq 0.6$ \\
\hline State 5 & $0.6 \leq \eta$ \\
\hline
\end{tabular}

In Eq. (10), i, j show the indices related to a sub-holon and its approaching links respectively. For example, 11,3 means the $3^{\text {rd }}$ approaching link of the 1 st sub-holon. Assume that the maximum density value of approaching links related to a sub-holon is $p$ :

$$
p=i \mid \exists i, j \rightarrow \quad l_{i, j}=\eta
$$

The action space of the super-holons is defined to restrict the autonomy of its sub-holons:

- Action 1: No command

- Action 2: Change the priority of $p$

- Action 3: Pre-emption of green time for $l_{i, j}=h$ such that the lowest green time is assigned to the other phases and the remaining green time is assigned to $l_{i, j}$.

- Action 4: Joint action for $\mathrm{p}$ and its neighboring subholon connected through $l_{i, j}=h$ called $q$ to change their priorities.

- Action 5: Joint action for $p$ and $q$, such that $p$ pre-empts the green time for $l_{i, j}$ and $q$ changes the priority of the opposite link of $l_{i, j}$ to higher priority. When the superholon selects Action 1, there is no command for sub- holons and therefore sub-holons can select an action autonomously Action. If the super-holon selects Action 2 or Action 4, sub-holons change the priority and select an action among the actions that satisfy the following equation instead of Eq. (9).

$$
x_{j}=2 ; \quad \sum_{i=1, i \neq j}^{4} x_{i}=2, \quad x_{i} \in\{0,1,2\}
$$

Pre-emption in 3 and Action 5 means that the subholons can select an action such that:

$$
x_{j}=4 ; \quad \forall x_{i}(i=1, \ldots, 4) \quad i \neq j \quad x_{i}=0
$$

\section{EXPERIMENTAL RESULTS}

The method has been tested on a network shown in Fig. 2 . In order to evaluate the robustness of the holonic system, we run the proposed method in two different experiments to see:

- Adaptability to changing environments: Whether the system still works when the input parameters of the environment change, or not.

- Robustness against connection interruption: How does the system work if a part of the connection between holons is damaged?

Three scenarios have been used in simulation: a) low volume, under-saturated conditions, b) high volume, under-saturated, and c) high volume, close to saturation. For the first scenario, 500 vehicles per hour are entered to the network from each entry points, which is given as traffic demand 1 in Tab. 2. For the second and third scenario, 750 and 1000 vehicle per hour are entered from the points, which are respectively related to traffic demand 2 and traffic demand 3 in Tab. 2. The holons are trained during 10 hours simulation.

Aimsun ${ }^{1}$ traffic simulator has been used to generate the network. The number of intersections and the number of entry points are 16. The network is composed of 80 links, the average length of the links being 850 kilometers. The number of lanes per link is 3 and the maximum allowed speed of the vehicle is $50 \mathrm{~km} / \mathrm{h}$. During the simulation time steps, new vehicles are generated as governed by the Poisson process at each entry point that stands on the boundary of the network. Poisson distribution is commonly used to describe a random process to model the number of vehicles arrived in a given duration of time [48]. The network configuration parameters are shown in Tab. 2.

In order to evaluate the robustness of the system, we use a random function to force disturbances in connections. In experiments, a part of connections is damaged during the learning process. We use $0 \%, 25 \%, 50 \%, 75 \%$ and $100 \%$ noise in experiments. It means that a connection will be damaged during the learning process with probability of $0,0.25,0.5,0.75$ and 1 , respectively. The experiment with $0 \%$ noise is equivalent to a complete holonic system in which there is full control of super-holons. The experiments with $100 \%$ noise are equivalent to a system

${ }^{1}$ www.Aimsun.com 
without super-holons in which the sub-holons control the signals without any superior control. The average delay time values computed over 10 independent runs for different traffic demands are shown in Figs. 4 to 6. Fig. 4 shows the average delay time for the first scenario. The fixed-timing method is also simulated for this scenario for comparing the results of the proposed method and fixedtime method. It can be seen that the proposed method outperforms the fixed-time method even in the presence of noises.

Table 2 Network configuration parameters

\begin{tabular}{|c|c|}
\hline Properties & Value \\
\hline number of intersections & 16 \\
number of links & 80 \\
the average length of links & $850 \mathrm{~m}$ \\
number of lanes per links & 3 \\
maximum speed & $50 \mathrm{~km} / \mathrm{h}$ \\
number of input/output centroid & 16 \\
arrival distribution & Poisson \\
simulation duration & 10 hour \\
traffic demand 1 & $8000 \mathrm{veh} / \mathrm{hour}$ \\
traffic demand 2 & $12000 \mathrm{veh} / \mathrm{hour}$ \\
traffic demand 3 & $16000 \mathrm{veh} / \mathrm{hour}$ \\
\hline
\end{tabular}

Table 3 Average delay time values $(\mathrm{sec} / \mathrm{km})$

\begin{tabular}{|c|c|c|c|}
\hline Noise & Traffic demand 1 & Traffic demand 2 & Traffic demand 3 \\
\hline $0 \%$ & 37.89 & 51.91 & 168.79 \\
$25 \%$ & 40.12 & 78.99 & 179.62 \\
$50 \%$ & 41.97 & 104.08 & 179.22 \\
$75 \%$ & 43.89 & 129.60 & 181.34 \\
$100 \%$ & 46.24 & 149.91 & 168.06 \\
\hline
\end{tabular}

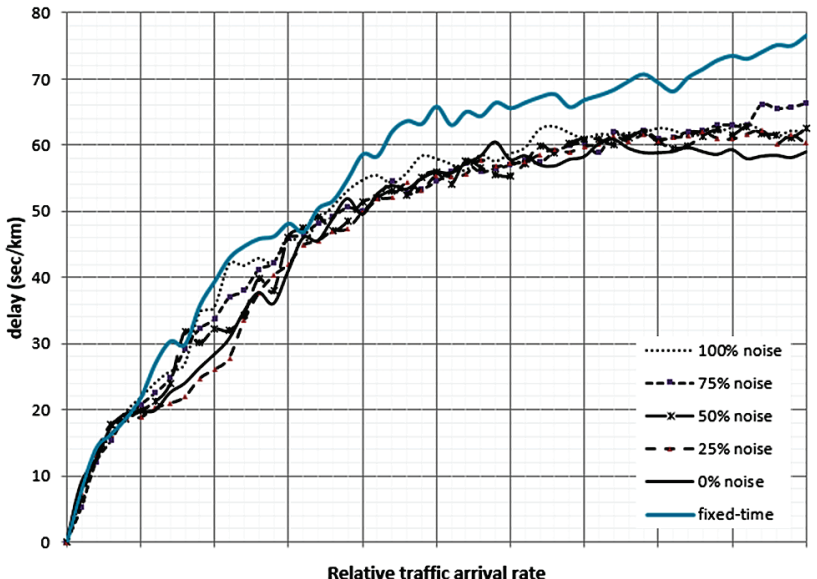

Figure 4 Average delay time of simulation along relative traffic arrival rate for traffic demand 1

The average delay time values computed over 10 simulations for the second traffic demand that is related to high volume, under-saturated conditions are shown in Fig. 5 .

Third traffic demand is related to high volume, close to saturation condition. It means that the number of vehicles entered to the network is more than the number of vehicles exited from the network and therefore some parts of the network will be full in such a way that it is not possible for any more vehicles to be entered to that part. The average delay time values computed over 10 simulations are shown in Fig. 6. It can be seen in the figure that although the network is saturated in all situations, the saturation time is different. The numerical delay values for different experiments are given in Tab. 3. The data reported in the table is not valid for traffic demand 3 , since the average values are computed before saturation time while it is not the same in all cases. Although the performance of a robust system decreases when it faces the disturbances, the decrement value can be estimated with respect to the disturbances. In other words, the behavior of the system can be estimated for a definite disturbance.

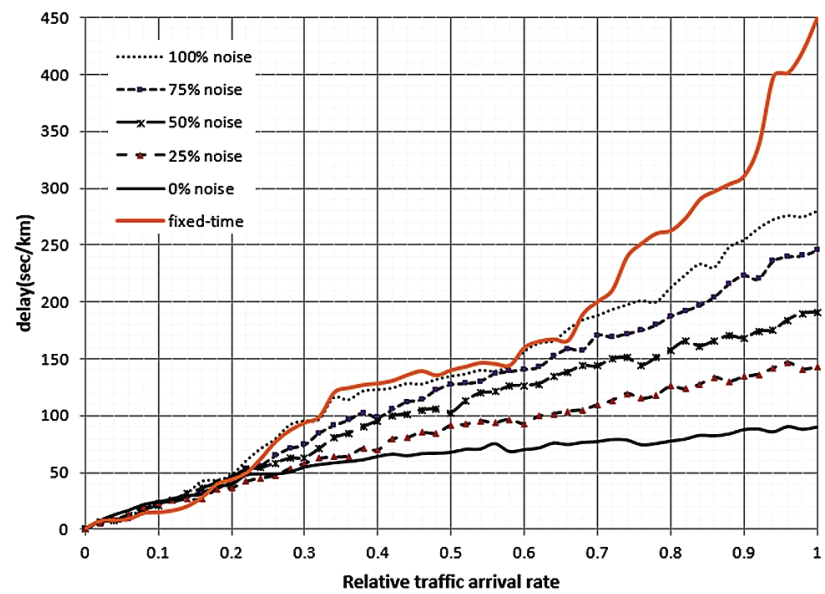

Figure 5 Average delay time of simulation according relative traffic arrival rate for traffic demand 2

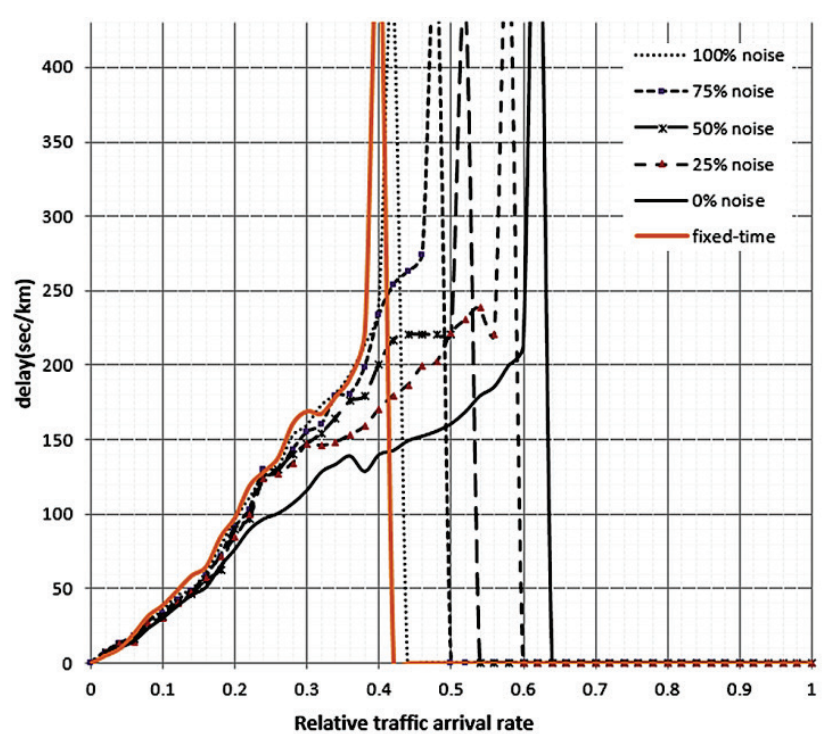

Figure 6 Average delay time of simulation according relative traffic arrival rate for traffic demand 3

The classic method for comparing two independent groups is Wilcoxon Mann-Whitney test which is derived by Wilcoxon in 1945. It is one of the most well-known nonparametric statistical hypothesis tests to determine whether one of two samples of independent observations tends to have larger values than other. Using the MannWhitney-Wilcoxon test, we can decide whether the population distributions are identical without assuming them to have a normal distribution. In order to show the robustness of the holonic traffic signal controllers, we compare the difference between the average delay times, when we increase the disturbances $25 \%$. According to Eq. 4 to Eq. (7) the value of $Z$ is computed when we increase the disturbances from $0 \%$ to $25 \%, 25 \%$ to $50 \%, 50 \%$ to $75 \%$, and $75 \%$ to $100 \%$. Tab. 4 shows the value of $Z$ computed for different conditions. In this table we use notations of $\mathrm{G}_{1}$ for $<0 \%, 25 \%>, \mathrm{G}_{2}$ for $<25 \%, 50 \%>, \mathrm{G}_{3}$ 
for $<50 \%, 75 \%>$ and $\mathrm{G}_{4}$ for $<75 \%, 100 \%>$. The value of $\mathrm{c}$ is $1.645,1.96$ and 2.58 for $\alpha=0.1, \alpha=0.05$ and $\alpha=0.01$ respectively [43].

Table 4 The value of $|\mathrm{Z}|$ computed for different disturbance rate. TD stands for traffic demand $i$

\begin{tabular}{|c|c|c|c|}
\hline \multicolumn{4}{|c|}{ traffic demand $i$} \\
\hline $\mathrm{G}_{1} \& \mathrm{G}_{2}$ & TD 1 & TD 2 & TD 3 \\
$\mathrm{G}_{2} \& \mathrm{G}_{3}$ & 0.71 & 1.21 & 1.70 \\
$\mathrm{G}_{3} \& \mathrm{G}_{4}$ & 0.84 & 1.53 & 1.84 \\
\end{tabular}

Table 5 Validity of Eq. (8) for different traffic demands

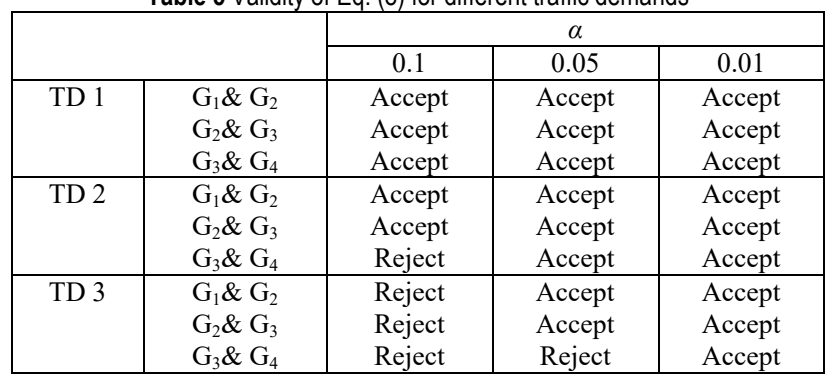

The validity of the proposition which is given in Eq. 8 with respect to different values of $\alpha$ is shown in Tab. 5 . When the traffic demand is low volume, for the first traffic demand, an equal increment in disturbances leads to an equal difference between average delay times in the simulation. As it can be seen in the table, for different values of $\alpha$ the distributions of the difference are identical. It shows the robustness of the proposed holonic system for low volume traffic demand. For high volume but under saturated traffic demand, it can be seen that the distributions are also identical when the disturbances increase $25 \%$. It shows that the proposed holonic system is robust for under saturated traffic demands. Fig. 6 shows that in high volume close to saturation traffic demand, the network starts to be saturated after some hours. Although in all conditions the saturation happens, the saturation time is different. The difference between the average delay times has identical distribution when the disturbance rate increases. Since the average delay time is measured before network saturation, the data collected in different disturbance rates are not the same. Therefore, we can conclude that even in close to saturation traffic demand, the system is robust.

\section{CONCLUSIONS AND FUTURE WORKS}

Robustness is essential for providing reliable solutions, able to be used in real world applications. The holonic organization offers advantages: they are robust in the face of external and internal disturbances, they are efficient in their use of resources, and they can adapt to environmental changes. Therefore, holonic organization provides a robust organization that can be used in distributed problems. In this paper, we have concentrated on the robustness of the holonic multi-agent system for traffic signals control. We showed that the holonic organization is robust for internal and external disturbances. The performance of the proposed method for internal disturbances, i.e. noise in communication, and for external disturbances, i.e. various traffic demands, shows that the holonic organization provides a robust solution for traffic signals control. Future works will include analysis of other features of holonic multi-agent system either in traffic signals control or other real-world applications, and present a robust holonic model for large-scale problems.

\section{REFERENCES}

[1] Theocharopoulou, C., Partsakoulakis, I., Vouros, G., \& Stergiou, K. (2007). Overlay Networks for Task Allocation and Coordination in Dynamic Large-scale Networks of Cooperative Agents. Proceeding of $6^{\text {th }}$ International Joint Conference on Autonomous Agents and Multi Agent Systems, p. 55. https://doi.org/10.1145/1329125.1329190

[2] Horling, B. \& Lesser V. (2004). A Survey of Multi-agent Organizational Paradigms. The Knowledge Engineering Review, 19(4), 281-316. https://doi.org/10.1017/S0269888905000317

[3] Chen, B. \& Cheng, H. (2010). A Review of the Applications of Agent Technology in Traffic and Transportation Systems. IEEE Transactions on Intelligent Transportation Systems, 11(2), 485-497. https://doi.org/10.1109/TITS.2010.2048313

[4] Bazzan, A. L. C. \& Klügl, F. (2014). A Review on Agentbased Technology for Traffic and Transportation. The Knowledge Engineering Review, 29(3), 375-403. https://doi.org/10.1017/S0269888913000118

[5] Chen, C. \& Li, Z. (2012). A Hierarchical Networked Urban Traffic Signal Control System Based on Multi-agent. $9^{\text {th }}$ IEEE International Conference on Networking, Sensing and Control (ICNSC), 28-33. https://doi.org/10.1109/ICNSC.2012.6204886

[6] Choy, M., Srinivasan, D., \& Cheu, R. (2003). Cooperative, Hybrid Agent Architecture for Real-time Traffic Signal Control. IEEE Transactions on Systems, Man and Cybernetics, Part A: Systems and Humans, 33(5), 597-607. https://doi.org/10.1109/TSMCA.2003.817394

[7] Srinivasan, D., Choy, M., \& Cheu, R. (2006). Neural Networks for Real-time Traffic Signal Control. IEEE Transactions on Intelligent Transportation Systems, 7(3), 261-272. https://doi.org/10.1109/TITS.2006.874716

[8] France, J. \& Ghorbani, A. (2003). A Multi-agent System for Optimizing Urban Traffic. Proceedings of the IEEE/WIC International Conference on Intelligent Agent Technology, Washington, DC, USA, 411-414.

[9] Bazzan, A. L. C., de Oliveira, D., \& da Silva, B. C. (2010). Learning in Groups of Traffic Signals. Engineering Applications of Artificial Intelligence, 23(4), 560-568. https://doi.org/10.1016/j.engappai.2009.11.009

[10] Wang, F. Y. (2005). Agent-based Control for Networked Traffic Management Systems. IEEE Intelligent Systems, 20(5), 92-96. https://doi.org/10.1109/MIS.2005.80

[11] Wang, F. (2008). Toward a Revolution in Transportation Operations: AI for Complex Systems. IEEE Intelligent Systems, 23(6), 813.

[12] Abdoos, M., Mozayani, N., \& Bazzan, A. L. C. (2011). Traffic Light Control in Nonstationary Environments Based on Multi-agent Q-learning. Proceeding of $14^{\text {th }}$ International Conference on Intelligent Transportation Systems, 580- 585.

[13] Touhbi, S. et al. (2017). Adaptive Traffic Signal Control: Exploring Reward Definition for Reinforcement Learning. Procedia Computer Science, 109, 513-520. https://doi.org/10.1016/j.procs.2017.05.327

[14] Genders, W. \& Razavi, S. (2016). Using a Deep Reinforcement Learning Agent for Traffic Signal Control. arXiv preprint arXiv: 1611.01142 .

[15] Mannion, P., Duggan, J., \& Howley, E. (2016). An Experimental Review of Reinforcement Learning Algorithms for Adaptive Traffic Signal Control. Autonomic Road Transport Support Systems, 47-66. https://doi.org/10.1007/978-3-319-25808-9_4 
[16] Bazzan, A. L. C. (2000). Opportunities for Multi-agent Systems and Multi-agent Reinforcement Learning in Traffic Control. Autonomous Agents and Multi-Agent Systems, 18(3), 342-375. https://doi.org/10.1007/s10458-008-9062-9

[17] Abdoos, M., Mozayani, N., \& Bazzan, A. L. C. (2014). Hierarchical Control of Traffic Signals Using Q-learning with Tile Coding. Applied Intelligence, 40(2), 201-213. https://doi.org/10.1007/s10489-013-0455-3

[18] Abdoos, M., Mozayani, N., \& Bazzan, A. L. C. (2015). Towards Reinforcement Learning for Holonic Multi-agent Systems. Intelligent Data Analysis, 19(2), 211-232.

[19] Abdoos, M., Mozayani, N., \& Bazzan, A. L. C. (2013). Holonic Multi-agent System for Traffic Signals Control. Engineering Applications of Artificial Intelligence, 26(5), 1575-1587. https://doi.org/10.1016/j.engappai.2013.01.007

[20] Koestler, A. (1967). The Ghost in the Machine, Hutchinson.

[21] Rodriguez, S., Gaud, N., \& Galland, S. (2014). SARL: A General-purpose Agent-oriented Programming Language. Proceedings of 2014 IEEE/WIC/ACM International Joint Conferences on Web Intelligence and Intelligent Agent Technologies / Poland, 103-110. https://doi.org/10.1109/WI-IAT.2014.156

[22] Mak, V., Schirrmann, A., Trentesaux, D., \& Vrba P. Industrial Applications of Holonic and Multi-Agent Systems, 2015

[23] Basiry, I. \& Ghasem-Aghaee, N. (2016). Crisis Control Mechanism in Holarchy Architecture. Journal of Knowledge-Based Engineering and Innovation, 2(4), 239249.

[24] Rodriguez, S. A., Hilaire, V., \& Koukam, A. (2005). Formal Specification of Holonic Multi-agent. Proceeding of International Conference on Computational Science, 719726.

[25] Benaskeur A. \& Irandoust, H. (2008). Holonic approach for control and coordination of distributed sensors. Technical report, DRDC Valcartier TR 2008-015, Defence R\&D Canada-Valcartier (Quebec), Canada.

[26] Rodriguez, S., Hilaire, V., \& Koukam, A. (2003). Towards a Methodological Framework for Holonic Multi-agent systems. Proceeding of Fourth International Workshop of Engineering Societies in the Agents World, 179-185.

[27] Marcellino, F. J. M. \& Sichman, J. (2010). A Holonic Multiagent Model for Oil Industry Supply Chain Management. Advances in Artificial Intelligence-IBERAMIA, 244-253. https://doi.org/10.1007/978-3-642-16952-6_25

[28] Ulieru, M. \& Geras, A. (2002). Emergent Holarchies for Ehealth Applications: a Case in Glaucoma Diagnosis. Proceeding of $28^{\text {th }}$ Annual Conference of the Industrial Electronics Society, 2957-2961. https://doi.org/10.1109/IECON.2002.1182866

[29] Shafaei, S. \& Aghaee, N. G. (2008). Biological Network Simulation Using Holonic Multi-agent Systems. Proceeding of $10^{\text {th }}$ International Conference on Computer Modeling and Simulation, 617-622.

[30] Moise, G. (2008). An Agent-holon Oriented Methodology to Build Complex Software Systems. International Journal of Computers, Communications, and Control, 3, 402-407.

[31] Akbari, A., Mozayani, N. (2014). A Holonic Multi Agent System for Operating Smart Grid Market. $4^{\text {rd }}$ Conference on Emerging Trends in Energy Conservation.

[32] Gaud, N., Gechter, F., Galland, S., \& Koukam, A. (2007). Holonic Multi-agent Multilevel Simulation Application to Real-time Pedestrians Simulation in Urban Environment. Proceedings of the $20^{\text {th }}$ International Joint Conference on Artificial Intelligence, 1275-1280.

[33] Versteegh, F., Salido, M., Giret, A. (2010). A Holonic Architecture for the Global Road Transportation System. Journal of Intelligent Manufacturing, 21(1), 133-144. https://doi.org/10.1007/s10845-008-0165-6
[34] Brckert, H., Fischer, K., \& Vierke, G. (2000). Holonic Transport Scheduling with Teletruck. Applied Artificial Intelligence, 14(7), 697-725. https://doi.org/10.1080/08839510050119253

[35] EOS. (2011). The Design. https://books.google.com/ books?id=3naHAwAAQBAJ by Lulu.com.

[36] Yang, X., Wang, J., \& Tan, Y. (2012). Robustness Analysis of Leader-follower Consensus for Multi-agent Systems Characterized by Double Integrators. Systems \& Control Letters, 61(11), 1103-1115. https://doi.org/10.1016/j.sysconle.2012.08.003

[37] Merdan, M., Moser, T., Vrba, P., \& Biffl, S. (2011). Investigating the Robustness of Re-scheduling Policies with Multi-agent System Simulation. The International Journal of Advanced Manufacturing Technology, 55(1-4), 355-367. https://doi.org/10.1007/s00170-010-3049-9

[38] Li, Z. \& Chen, J. (2016). Robust Consensus of Multi-agent Systems with Stochastic Uncertain Channels. In American Control Conference (ACC), 3722-3727.

[39] Wen, G., Hu, G., Zuo, Z., Zhao, Y., \& Cao, J. (2017). Robust Containment of Uncertain Linear Multi-agent Systems under Adaptive Protocols. International Journal of Robust and Nonlinear Control, 27(12), 2053-2069. https://doi.org/10.1002/rnc.3670

[40] Belgacem, S. \& Fates, N. (2012). Robustness of Multi-agent Models: the Example of Collaboration between Turmites with Synchronous and Asynchronous Updating. Complex Systems, 21(3), 165-182.

[41] Snyder, R. D., MacKenzie, D. C., \& Tomlinson, R. S. (2004). Robustness Infrastructure for Multi-agent Systems. In Proceedings of the $1^{\text {st }}$ International Conference Open Cougaar, 75-80.

[42] Leitao, P., Valckenaers, P., \& Adam, E. (2009). Selfadaptation for Robustness and Cooperation in Holonic Multi-agent Systems. Transactions on Large-Scale Dataand Knowledge-Centred Systems I, 267- 288. https://doi.org/10.1007/978-3-642-03722-1_11

[43] Khalil, H. \& Grizzle, J. (1996). Nonlinear Systems, Prentice - Hall, Inc. New Jersey.

[44] Bracciali, A., Mancarella, P., Stathis, K., \& Toni, F. (2004). Engineering Stable Multi-agent Systems. International Workshop on Engineering Societies in the Agent World, 322334

[45] Chli, M. et al. (2003). Stability of Multi-agent Systems IEEE International Conference on Systems, Man and Cybernetics, 551-556. https://doi.org/10.1109/ICSMC.2003.1243872

[46] Jen, E. (2003). Stable or Robust? What's the Difference? Complexity, 8(3), 12-18. https://doi.org/10.1002/cplx.10077

[47] Wilcox, R. (2009). Basic Statistics: Understanding Conventional Methods and Modern Insights, Oxford University Press.

[48] Adolf, D. M. (1990). Fundamentals of Traffic Flow. Prentice - Hall, Inc. Englewood Cliff New Jersey 07632, second edition.

\section{Contact information:}

Monireh ABDO0S, Assistant Professor

Faculty of Computer Science and Engineering,

Shahid Beheshti University,

Velenjak, 19839-63113 Tehran, Iran

m_abdoos@sbu.ac.ir 